\title{
Publisher's Note: Experimental demonstration of a global dispersion-free steering correction at the new linac test facility at SLAC [Phys. Rev. ST Accel. Beams 17, 042803 (2014)]
}

\author{
A. Latina, J. Pfingstner, D. Schulte, E. Adli, F. J. Decker, and N. Lipkowitz \\ (Received 30 April 2014; published 5 May 2014)
}

DOI: 10.1103/PhysRevSTAB.17.059901

PACS numbers: 29.20.Ej, 29.27.Eg, 99.10.Fg

This paper was published online on 11 April 2014 with an error in the text of the caption to Fig. 8. The fourth sentence of the figure caption should read as "Note that the absolute orbit is not necessarily improved as the dispersion is reduced...." The figure caption has been corrected online as of 1 May 2014. 